\title{
INKJET PRINTING OF SU-8 FOR POLYMER-BASED MEMS A CASE STUDY FOR MICROLENSES V. Fakhfouri ${ }^{1}$, N. Cantale $^{2}$, G. Mermoud ${ }^{3}$, J.Y. Kim ${ }^{1}$, D. Boiko ${ }^{2}$, E. Charbon ${ }^{2}$, A. Martinoli ${ }^{3}$ and J. Brugger ${ }^{1}$ \\ ${ }^{1,2,3}$ École Polytechnique Fédérale de Lausanne (EPFL), Lausanne, Switzerland \\ ${ }^{1}$ Microsystems Laboratory \\ ${ }^{2}$ Quantum Architecture Group \\ ${ }^{3}$ Swarm-Intelligent Systems Group
}

\begin{abstract}
This paper describes a novel method to fabricate polymer MEMS based on the inkjet printing of SU-8, with a special emphasis on integrated micro-optical lens arrays. Inkjet control parameters are optimized in order to enable a stable and reproducible ejection of SU-8 drops in both continuous and drop-on-demand (DOD) modes. Arbitrary patterns of single and multiple polymer drops and arrays of convex microlenses are printed on different substrates. The influence of surface wetting properties on the size and the shape of the printed patterns is investigated. The optical properties of the microlenses are investigated in details. A model for inkjet printing of high-viscous functional materials for polymer MEMS has been used.
\end{abstract}

\section{INTRODUCTION}

Since its first introduction to MEMS in 1997 [1], SU-8 has enormously contributed to the advances of MEMS, either as high aspect-ratio mold or as permanent material [2]. A high transparency for wavelengths $>400 \mathrm{~nm}$ makes SU-8 a suitable material for integrated optics applications [3]. Complementary to photolithography, inkjet printing is increasingly considered a cost-effective and flexible method for the fabrication of functional materials [4] such as conducting polymers [5]. The ease of mass fabrication and the inherent flexibility of inkjet technology makes it a suitable method for the manufacturing of polymer lightemitting diodes [6]. Microlenses are typically fabricated using different techniques, such as manufacturing by melting photoresist [7] and transfer into fused silica and silicon. Inkjet printing of silica based inorganic-organic material has also been used as a post-processing technique for the fabrication of refractive microlens arrays [8].

Here we present a further expansion of the versatility and applicability of polymer MEMS by DOD inkjet printing of SU-8. We focus on the printing of microlenses using a commercially available material and provide a model that allows expanding the method towards other applications in the field of polymer MEMS.

\section{THEORY OF DROP GENERATION}

A thorough understanding of the underlying mechanism of inkjet printing of functional materials with viscosities in the range of $30-150 \mathrm{mPa}$ s was necessary to determine and optimize the print parameters in view of a reliable jet conditions. Each different control parameter of the piezoactuated printer head plays a role for the DOD generation of
SU-8 drops.

Numerous studies show that for both steady and transient flows, the onset and mechanisms of droplet breakup (the main condition to generate a drop) can be correlated with the dimensionless Weber number, $\mathrm{We}$ [9]:

$$
W e=d v_{g}^{2} \rho \sigma_{l}^{-1}
$$

In this equation $d$ is the drops' diameter, $v_{g}$ is the relative velocity between the gas (air in our case) and the drop, $\rho$ is the density and $\sigma_{l}$ is the surface tension of the liquid. As categorized in previous works [10], no breakup occurs for liquids like water when $W e<12$. Increasing the liquid viscosity delays the onset of breakup, resulting in a different critical Weber number below which breakup does not occur. The corresponding dimensionless parameter is the Ohnesorge number:

$$
O n=\mu_{l}\left(\rho d \sigma_{l}\right)^{-0.5}
$$

Here $\mu_{l}$ and $\rho$ are the dynamic viscosity and the density of the liquid. This number describes the materials' properties and the size of the drop (this corresponds to the size of the nozzle orifice in our case). The critical Weber number for high viscous fluids is given by:

$$
W e_{\text {critical }}=12\left(1-1.077 \cdot \mathrm{On}^{1.6}\right)
$$

Our objective is to determine the critical and the maximum Weber numbers necessary to generate single drops without satellites. The optimal number allows us to determine the inkjet velocity range suitable for materials with similar properties.

\section{EXPERIMENTAL TECHNIQUES Inkjet printing}

We use a piezo-actuated inkjet printer head (microdrop technologies, Germany) with a nozzle aperture of $50 \mu \mathrm{m}$ with a heated nozzle tip to inkjet print SU-8 (SU-8 based negative tone photoresist provided by micro resist technology, Germany). The microdrops are observed via a stroboscopic vision system. The amplitude and the length of the signal sent to the piezo-actuator as well as the nozzle temperature are controlled. A computer-controlled $x-y-\theta$ stage allows the positioning of the sample with respect to 
the impact location of the droplet. Patterns made of single or multiple drops of SU-8 are created either by continuous or drop-on-demand (DOD) mode. In the first mode, the drop ejection frequency, stage scan speed and dwell time determine the shape of the final pattern. The DOD mode enables the fabrication of fully arbitrary patterns, because the droplets can be ejected individually at arbitrary times and positions. Furthermore the alignment of the nozzle to pre-structured substrates is feasible in this mode. After deposition, the drops are prebaked $\left(100^{\circ} \mathrm{C}, 15\right.$ minutes), UV-exposed $\left(400 \mathrm{~mJ} / \mathrm{cm}^{2}\right)$ and post-baked $\left(100^{\circ} \mathrm{C}, 15\right.$ minutes).

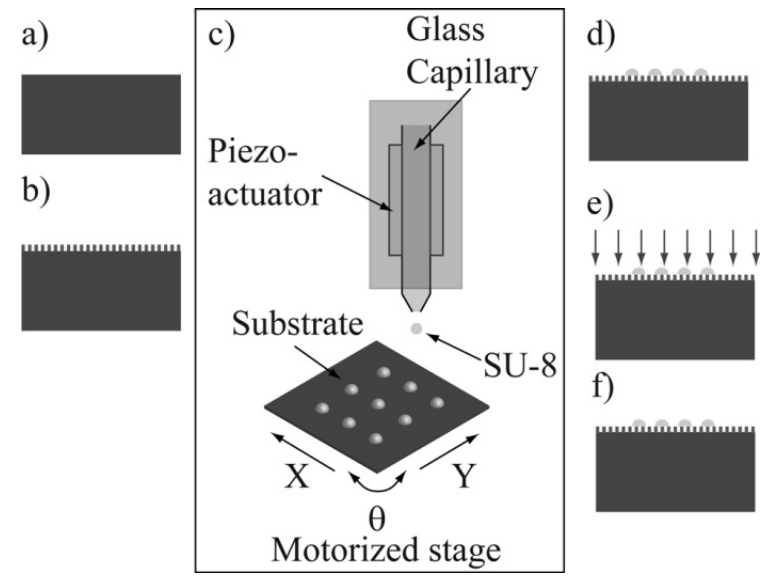

Figure 1: Schematic illustration of the experimental procedure: a) substrate preparation, b) surface functionalisation (e.g. SAM treatment, coating), c) inkjet printing, d) prebake, e) UV-exposure, f) post exposure bake

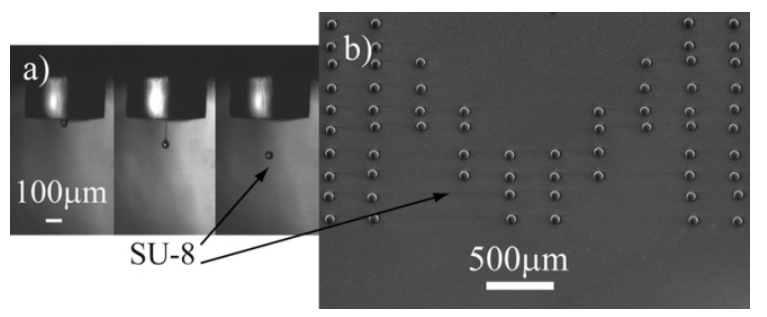

Figure 2: a) Sequence of stroboscopic images of SU8 drop generation, 100, 200 and $300 \mu$ s after the generation of the pulse, b) SEM image of an array of $S U-8$ single drops forming the letter M on SAMcoated glass

\section{Imaging of the $\mathrm{SU}-8$ microlenses}

The microlenses are imaged using an optical microscope and an image processing algorithm using Sobel filtering and Morphometry analysis automatically measures the lens diameter on different substrates. A Scanning Electron Microscope (SEM) and a Digital Holographic Microscope (DHM) are used to inspect the 3D shape of the microlenses, which are coated by a $20 \mathrm{~nm}$-thick layer of gold for that purpose.
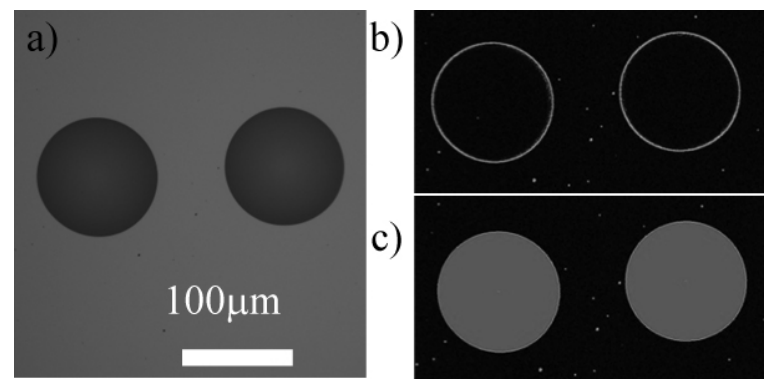

Figure 3: Image processing algorithm: a) optical microscopy image of a SU-8 drop on silicon, b) Sobel filtered image, c) Morphometry analysis including the size and the elliptical form factor measurement

\section{Optical characterization}

In view of micro-optical applications two characterization methods are used to measure the diameter, the beam spot diameter at the focal plane (the Airy disk diameter), the focal length and the numerical aperture.

In the first method, the microlenses are illuminated from the convex side using a He-Ne laser. The microlenses or their back focal plane are imaged onto a CCD camera. This image is used to extract the shape of the spot at the focal plane of a microlens. Tuning the position of the microlenses from the location corresponding to the imaging of the back focal plan to the position for imaging a microlens itself will allow us to measure the focal distance. Secondly, a Mach-Zehnder interferometer has been used to determine the focal length and the numerical aperture as well as the aberration and the Strehl ratio, for which the lenses are illuminated from the flat side.
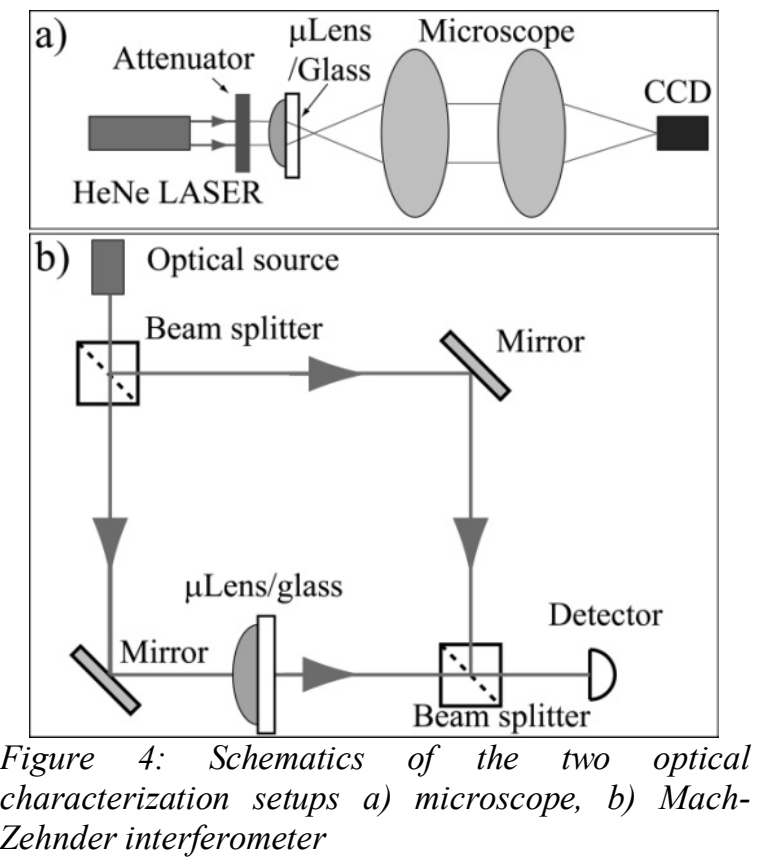


\section{RESULTS}

\section{Inkjet printing parameters}

The variation of the drops' velocity as a function of the inkjet printing parameters, namely the pulse length and amplitude and the temperature of the nozzle have been measured to determine the minimum, the maximum and the optimal Weber numbers for SU-8. These values have been determined by observing the displacement of the drop using the stroboscopic vision system.

The optimum inkjet parameters obtained experimentally correspond to the cases where we obtained stable generation of the drops, without any satellite-drop generation. These values and the maximum and minimum acceptable velocities are shown in the figures below.

We have measured $7.95 \mathrm{~mm}^{2} / \mathrm{s}$ for the kinematic viscosity of SU-8 (formulation provided by micro resist) at the optimal temperature (between 60 and $90^{\circ} \mathrm{C}$ ) using a capillary viscometer in order to derive the optimal dynamic viscosity. We assume the density value of $1.123 \mathrm{~g} / \mathrm{cm}^{3}$ and a surface tension of about $48 \mathrm{mN} / \mathrm{m}$ (data provided by micro resist technology) to calculate a Weber number of 4.68 for a velocity of $2 \mathrm{~m} / \mathrm{s}$ for a stable inkjet of SU-8 in both continuous and DOD modes. The corresponding Ohnesorge number is 0.17 for a critical Weber number of 11.2.

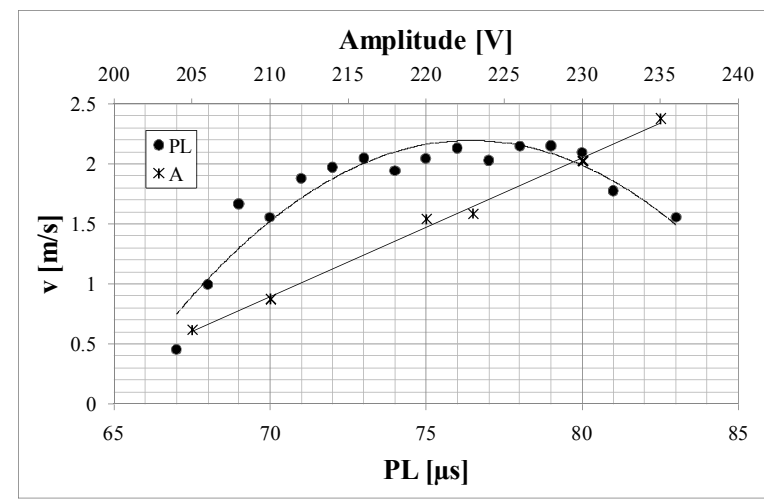

Figure 5: Drop velocity versus pulse amplitude (A) and pulse length $(P L)$

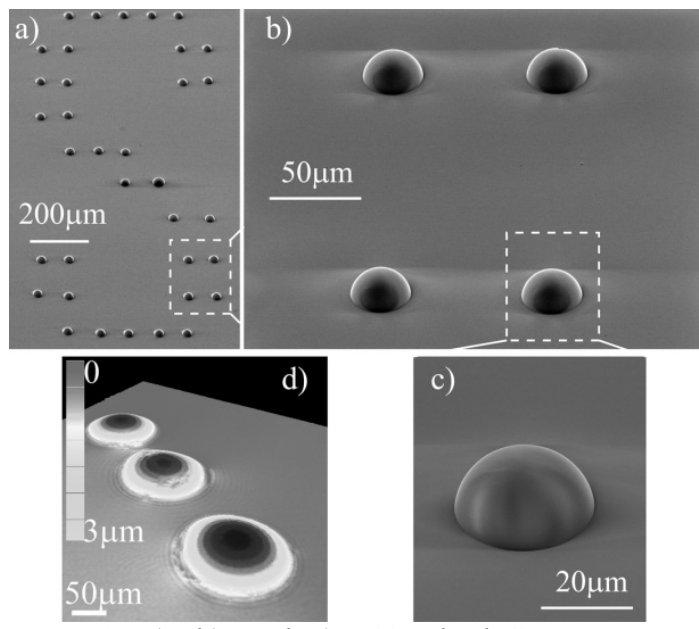

Figure 6: a), b) and c) $50^{\circ}$ tilted SEM images of DOD inkjet-printed patterns of SU-8 on SAM-coated glass, d) DHM image of SU-8 drops on untreated $\mathrm{Si}$

\section{Shape and size of microlenses}

The shape of the SU-8 microlenses depends on the surface wetting properties. The mean value of the radius of a single SU-8 drop on the tested surfaces varies from $29 \mu \mathrm{m}$ to $71 \mu \mathrm{m}$ depending on the surface (Table 1) showing the possibility of tuning the lens characteristics by appropriate surface coatings.

\begin{tabular}{|l|c|c|}
\hline \multicolumn{1}{|c|}{ Substrate } & $\begin{array}{c}\text { Mean radius } \\
{[\boldsymbol{\mu \mathbf { m } ]}]}\end{array}$ & $\begin{array}{c}\text { Standard } \\
\text { deviation } \\
{[\boldsymbol{\mu \mathbf { m } ]}}\end{array}$ \\
\hline Si (untreated) & 58.5 & 0.4 \\
\hline Glass (untreated) & 71.4 & 0.1 \\
\hline Quartz (untreated) & 59.7 & 0.04 \\
\hline $\begin{array}{l}\text { Teflon-like coated Si } \\
\text { (110nm) }\end{array}$ & 55.9 & 0.2 \\
\hline $\begin{array}{l}\text { Teflon-like coated Si } \\
\text { (240nm) }\end{array}$ & 55.1 & 0.8 \\
\hline SAM coated Si & 28.9 & 0.08 \\
\hline
\end{tabular}

Table 1: Comparison of the lens radius on different substrates based on 30 measurements per substrate

The 3D shape of the microlenses has been investigated by tilted SEM imaging. As anticipated and shown in Fig 7, a significant increase in droplet height occurred in the case of the substrates coated with the SAM layer.

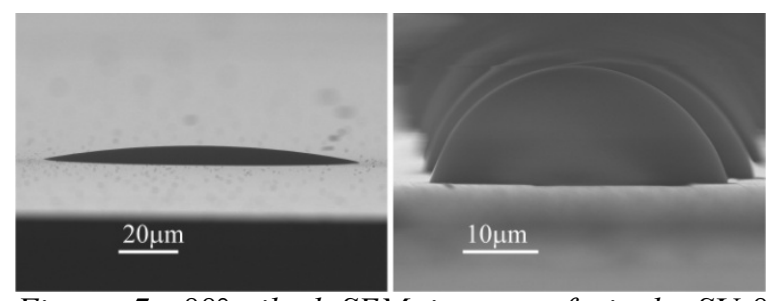

Figure 7: $88^{\circ}$ tilted SEM images of single SU-8

drops: left, on silicon; right, on SAM-coated silicon

\section{Optical characteristics}

The results for 3 different SU-8 microlens arrays (of 19, 39 and 9 microlenses) deposited on untreated glass surfaces show that the mean focal lengths are $78.6 \pm 14.9,99.8 \pm 9.8$ and $120.4 \pm 26.2 \mu \mathrm{m}$ respectively and are within the range of interest for miniaturized imaging systems [11]. Furthermore the values measured for the spot diameter are close to the diffraction-limited Airy disk size indicating the high quality of the inkjet-printed microlenses.

\begin{tabular}{|l|l|l|l|l|}
\hline $\begin{array}{l}\text { Focal } \\
\text { length } \\
{[\mu \mathrm{m}]}\end{array}$ & $\begin{array}{l}\text { Airy disk } \\
\text { diameter } \\
{[\mu \mathrm{m}]}\end{array}$ & $\begin{array}{l}\text { Estimated } \\
\text { diffraction- } \\
\text { limited spot } \\
\text { diameter }\end{array}$ & $\begin{array}{l}\text { A.D. / } \\
\text { Diff. } \\
\text { Lim. }\end{array}$ & N.A. \\
\hline $55-153$ & $1.93-3.59$ & $1.05-2.09$ & $\begin{array}{l}1.17- \\
3.14\end{array}$ & $\begin{array}{l}0.36- \\
0.73\end{array}$ \\
\hline Table 2: & Ranges of optical characteristics of SU-8
\end{tabular}

microlenses deposited on untreated glass 


\begin{tabular}{|l|l|c|c|}
\cline { 3 - 4 } \multicolumn{2}{|c|}{} & Average & $\begin{array}{l}\text { Standard } \\
\text { deviation }\end{array}$ \\
\hline $\begin{array}{l}\text { Single drop } \\
\mu-\text {-lenses }\end{array}$ & $\mathbf{f}[\mu \mathrm{m}]$ & 41.9 & 1.68 \\
\cline { 2 - 4 } & NA & 0.46 & 0.02 \\
\hline $\begin{array}{l}\text { Double } \\
\text { drops } \\
\mu-\text {-lenses }\end{array}$ & $\mathbf{f}[\mu \mathrm{m}]$ & 73 & 1.5 \\
\cline { 2 - 4 } & NA & 0.25 & 0.01 \\
\hline
\end{tabular}

Table 3: Optical characteristics of SU-8 microlenses deposited on SAM coated glass

Further, we analyzed high-aspect ratio lenses made on the SAM-coated glass by Mach-Zehnder interferometry of 17 lenses made of single drops and 10 lenses made of 2 drops each (Table 3). The results show that the SAM layer improves the focal length uniformity of the inkjet-printed microlenses.

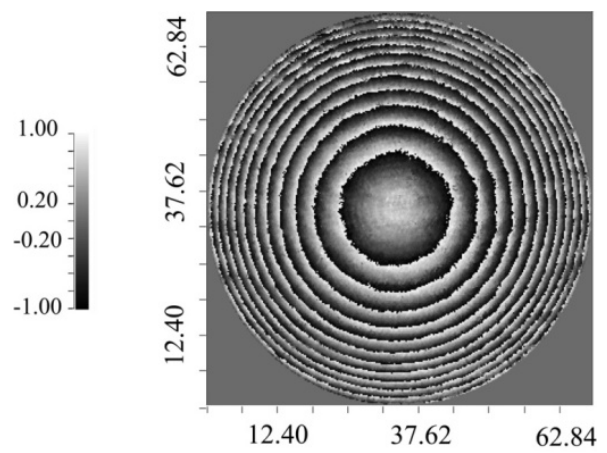

Figure 8: Phase modulation 2 $\pi / \pi$ Mach-Zehnder interferometry image of a typical microlens

\section{CONCLUSIONS AND OUTLOOK}

We have explored new possibilities of the inkjet printing technology for direct patterning of SU-8. We have presented our understanding of the mechanism of inkjet printing of materials with viscosities in the range of 30$150 \mathrm{mPa}$ a and determined the optimal parameters of inkjet printing for SU-8. Further investigations are ongoing to describe the detailed mechanism of inkjet printing of SU-8 based materials for other applications [12]

Arbitrary SU-8 drop patterns have been printed on different surfaces and their shape has been investigated. Microlenses for have been characterized and show interesting properties for micro-optical applications.. We consider the inkjet printing technology as a versatile and cost-effective technique for direct printing of functional materials and polymers, such as SU-8. Detailed studies of the behavior of the droplets after deposition and during the curing step, and the use of other polymers and composites are issues for future work.

\section{ACKNOWLEDGMENTS}

We thank B. Putz, Dr. K.J. Weible and Dr. R. Völkel of SUSS MicroOptics SA in Neuchâtel, Switzerland, for their precious help on characterizing the microlenses. We are also pleased to acknowledge the contribution of our colleague
J.C. Sarria who developed the image processing algorithms. Many thanks to Dr. A. Voigt and G. Grützner from micro resist technology for their help on material characterizations.

Part of this work is funded by the FP6-NMP Project "Novopoly", reference STRP 013619. We also acknowledge the "Centre SI" at EPFL for their support.

\section{REFERENCES}

[1] H. Lorenz, M. Despont, N. Fahrni, N. LaBianca, P. Renaud and P. Vettiger, "SU-8: a low-cost negative resist for MEMS", Journal of Micromechanics and Microengineering, 1997, (3), pp. 121-124

[2] G. Genolet, J. Brugger, M. Despont, U. Drechsler, P. Vettiger, N. F. de Rooij and D. Anselmetti, "Soft, entirely photoplastic probes for scanning force microscopy", Review of Scientific Instruments, 1999, (5), pp. 2398-2401

[3] B. Beche, N. Pelletier, E. Gaviot and J. Zyss, "Singlemode TE00-TM00 optical waveguides on SU-8 polymer", Optics Communications, 2004, (1-3), pp. 91-94

[4] B.-J. De Gans, U. S. Schubert and P. C. Duineveld, "Inkjet printing of polymers: State of the art and future developments", Advanced Materials, 2004, (3), pp. 203-213

[5] D. Pede, G. Serra and D. De Rossi, "Microfabrication of conducting polymer devices by ink-jet stereolithography", Materials Science \& Engineering C-Biomimetic and Supramolecular Systems, 1998, (34), pp. 289-291

[6] E. I. Haskal, H. J. Bolink, M. Büchel, P. C. Duineveld, B. Jacobs, M. M. De Kok, E. A. Meulenkamp, E. H. J. Schreurs, S. I. E. Vulto, P. Van de Weijer and S. H. P. M. De Winter, "Passive-matrix polymer light-emitting displays", Journal of the Society for Information Display, 2003, (1 SPEC.), pp. 155-160

[7] D. Daly, R. F. Stevens, M. C. Hutley and N. Davies, "The Manufacture of Microlenses by Melting Photoresist", Measurement Science \& Technology, 1990, (8), pp. 759-766

[8] R. Danzebrink and M. A. Aegerter, "Deposition of optical microlens arrays by ink-jet processes", Thin Solid Films, 2001, (2), pp. 223-225

[9] G. O. Thomas, "The aerodynamic breakup of ligaments", Atomization and Sprays, 2003, (1), pp. $117-129$

[10] M. Pilch and C. A. Erdman, "Use of Breakup Time Data and Velocity History Data to Predict the Maximum Size of Stable Fragments for AccelerationInduced Breakup of a Liquid-Drop", International Journal of Multiphase Flow, 1987, (6), pp. 741-757

[11] R. Völkel, M. Eisner and K. J. Weible, "Miniaturized imaging systems", Microelectronic Engineering, 2003, pp. $461-472$

[12] V. Fakhfouri, et. al., "Inkjet printing of high-viscous materials", manuscript in preparation 